\title{
TOPOLOGICALLY EQUIVALENT MEASURES IN THE CANTOR SPACE
}

\author{
FRANCISCO J. NAVARRO-BERMÚDEZ
}

\begin{abstract}
The Cantor space is realized as a countable product $X$ of two-element sets. The measures $\mu$ and $\nu$ in $X$ are topologically equivalent if there is a homeomorphism $h$ of $X$ onto itself such that $\mu=\nu h$. Let $\mathscr{F}$ be the family of product measures in $X$ which are shift invariant. The members $\mu(r)$ of $\mathscr{F}$ are in one-to-one correspondence with the real numbers $r$ in the unit interval. The relation of topological equivalence partitions the family $\mathscr{F}$ into classes with at most countably many measures each. A class contains only the measures $\mu(r)$ and $\mu(1-r)$ when $r$ is a rational or a transcendental number. Equivalently, if $r$ is rational or transcendental and $\mu(s)$ is topologically equivalent to $\mu(r)$ then $s=r$ or $s=1-r$.
\end{abstract}

1. Introduction. Let $X$ denote a topological space. Two Borel measures $\mu$ and $\nu$ in $X$ are said to be topologically equivalent whenever $\mu=\nu h$ for some homeomorphism $h$ of $X$ onto itself. This notion sets up an equivalence relation which partitions the family of Borel measures into disjoint classes, each one consisting of those measures which are mutually equivalent. By restricting attention, if necessary, to a suitably defined subfamily of measures, one can ask for the number of classes there are. One can also try to uncover necessary conditions, as well as sufficient conditions, which intrinsically characterize those measures which belong to the same class.

Topologically equivalent measures in the $n$-dimensional unit cube, the space of irrational numbers in the unit interval, and the Hilbert cube have been studied, respectively, by Oxtoby and Ulam [1], Oxtoby [2], and by Oxtoby and Prasad [3]. In this article I initiate the study of topologically equivalent measures in the Cantor space. By restricting attention to a suitably defined family of Borel measures, which, incidentally, are everywhere positive, nonatomic and normalized, I am able to utilize arguments of a number theoretic character and will be able to show, in particular, that the relation of topological equivalence partitions that family into a number of classes which is the same as the cardinality of the continuum.

2. Topological and measure theoretic preliminaries. It is well known that if $C$ is the Cantor "middle thirds" set and $X$ a nonempty, compact, perfect, 0 -dimensional metric space, there is a homeomorphism from $C$ onto $X$. In particular, $X$ can be taken to have the form

Presented to the Society, March 21, 1977; received by the editors August 30, 1978.

AMS (MOS) subject classifications (1970). Primary 28A35; Secondary 10A10.

(c) 1979 American Mathematical Society 0002-9939/79/0000-0513/\$03.00 


$$
X=\prod_{n=1}^{\infty} S_{n}
$$

where $S_{n}=\{1,2\}$ for all $n$. $X$ carries the product topology induced by the discrete topology in each of the factors $S_{n}$.

Every open set $U$ of $X$ is a union $\mathscr{U}$ of basic open sets. If $U$ is also closed, hence compact, then only finitely many basic open sets enter into that union. A basic open set is obtained by fixing finitely many coordinates. Thus, if the union $\mathscr{U}$ consists of only finitely many basic open sets, then there is a smallest positive integer $n$ such that none of the coordinates after the $n$th place is fixed in any of the basic open sets of the union $\mathscr{U}$. If the $n$th coordinate, or an earlier one, is not fixed in some basic open set of $\mathcal{Q}$, then it is possible to express this set as the union of two sets where that coordinate is fixed. For example, the set

$$
S_{1} \times\{2\} \times \cdots \times\{1\} \times S_{n+1} \times \cdots
$$

is the union of the two sets

$$
\{1\} \times\{2\} \times \cdots \times\{1\} \times S_{n+1} \times \ldots
$$

and

$$
\{2\} \times\{2\} \times \cdots \times\{1\} \times S_{n+1} \times \ldots .
$$

It follows that the closed-open set $U$ can be expressed as the union of finitely many disjoint basic open sets each of which has the form

$$
\left\{i_{1}\right\} \times\left\{i_{2}\right\} \times \cdots \times\left\{i_{n}\right\} \times S_{n+1} \times \ldots \quad\left(i_{k}=1 \text { or } 2\right)
$$

with the same $n$ for all of them.

Denote the set $(2.1)$ with the symbol $\left\langle i_{1}, i_{2}, \ldots, i_{n}\right\rangle$. Two of these sets $\left\langle i_{1}, i_{2}, \ldots, i_{n}\right\rangle$ and $\left\langle j_{1}, j_{2}, \ldots, j_{n}\right\rangle$ are equal or disjoint, depending on whether the $n$-tuples $\left(i_{1}, i_{2}, \ldots, i_{n}\right)$ and $\left(j_{1}, j_{2}, \ldots, j_{n}\right)$ are identical or not. Thus, the complement of $\left\langle i_{1}, i_{2}, \ldots, i_{n}\right\rangle$ is the union of all the sets $\left\langle k_{1}, k_{2}, \ldots, k_{n}\right\rangle$ where $\left(k_{1}, k_{2}, \ldots, k_{n}\right)$ is an $n$-tuple different from $\left(i_{1}, i_{2}, \ldots, i_{n}\right)$. This complement is an open set. Hence, the basic open set $\left\langle i_{1}, i_{2}, \ldots, i_{n}\right\rangle$ is closed as well. The sets $(2.1)$ will be called special closedopen sets of length $n .^{1}$ They are defined by the fact that all coordinates up to the $n$th place are fixed. The truth of the following theorem is seen immediately.

THEOREM 2.1. Let $U$ be a closed-open set of the Cantor space $X$. There is a positive integer $n$ such that $U$ can be expressed as the disjoint union of finitely many special closed-open sets of length $n$.

The family $\mathscr{F}$ of measures in $X$ to which attention will be restricted consists of product measures $\mu=\prod_{n=1}^{\infty} \mu_{n}$ subject to the condition

\footnotetext{
1 This terminology is not standard. Some authors call them "thin cylinders"; others call them "intervals". For the important computations in this paper (i.e., Theorem 2.2, and its consequences) they are crucial, hence my choice to call them "special".
} 


$$
\mu_{n}(1)=\mu_{n+1}(1) \text { for all } n \text {. }
$$

Of course, the factors $\mu_{n}$ are all normalized, and thus, as $\mu_{n}(1)+\mu_{n}(2)=1$, the same requirement $(2.2)$ holds with 1 replaced by 2 . For convenience write $\mu=\mu(r)$, where $r$ is the value $\mu_{n}(1)$. Amongst the product measures these are precisely those which are invariant under the shift transformation $T$ of $X$ defined by $T\left(x_{1}, x_{2}, x_{3}, \ldots\right)=\left(x_{2}, x_{3}, \ldots\right)$ for every point $x=$ $\left(x_{1}, x_{2}, x_{3}, \ldots\right)$.

For each real number $r$ in the unit interval there is a measure $\mu(r)$. As the correspondence $r \rightarrow \mu(r)$ is one-to-one, the family $\mathscr{F}$ has the cardinality of the continuum. Incidentally, each $\mu(r)$ is a normalized Borel measure which, except when $r$ equals 0 or 1 , is everywhere positive and nonatomic.

Let $U=\left\langle i_{1}, i_{2}, \ldots, i_{n}\right\rangle$ be a special closed-open set of length $n$. Then

$$
\mu(r)(U)=\mu_{1}\left(i_{1}\right) \mu_{2}\left(i_{2}\right) \ldots \mu_{n}\left(i_{n}\right) \text {, }
$$

and since $\mu_{k}\left(i_{k}\right)$ equals $r$ or $1-r$ depending on whether $i_{k}$ is 1 or 2 , it follows that

$$
\mu(r)(U)=r^{j}(1-r)^{n-j}
$$

where $j$ is the number of times that $i_{k}=1(1 \leqslant k \leqslant n)$.

It is evident that the measures $\mu(r)$ and $\mu(1-r)$ are always topologically equivalent, for, indeed, $\mu(1-r)=\mu(r) h$, where $h$ is the homeomorphism $h=\prod_{n=1}^{\infty} h_{n}$ with $h_{n}: S_{n} \rightarrow S_{n}$ given by $h_{n}(1)=2$ and $h_{n}(2)=1$ for all $n$.

THEOREM 2.2. If the measures $\mu(r)$ and $\mu(s)$ are topologically equivalent, then there exist positive integers $n$ and $m$, and integers $a_{0}, a_{1}, \ldots, a_{n}, b_{0}, b_{1}, \ldots, b_{m}$ with

$$
0 \leqslant a_{j} \leqslant n ! / j !(n-j) ! \text { and } 0 \leqslant b_{j} \leqslant m ! / j !(m-j) !
$$

such that

$$
s=a_{0} r^{n}+a_{1} r^{n-1}(1-r)+\cdots+a_{n-1} r(1-r)^{n-1}+a_{n}(1-r)^{n}
$$

and

$$
r=b_{0} s^{m}+b_{1} s^{m-1}(1-s)+\cdots+b_{m-1} s(1-s)^{m-1}+b_{m}(1-s)^{m} .
$$

Proof. Let $h$ be a homeomorphism of $X$ onto itself such that $\mu(s)=\mu(r) h$, and let $V$ denote the closed-open set $\langle 1\rangle$. On the one hand, $\mu(s)(V)=s$. On the other hand, $h(V)$, being a closed-open set is, by Theorem 2.1, a disjoint union $\mathcal{Q}$ of finitely many special closed-open sets $\left\langle i_{1}, i_{2}, \ldots, i_{n}\right\rangle$ of length $n$. By (2.3) the $\mu(r)$-measure of $\left\langle i_{1}, i_{2}, \ldots, i_{n}\right\rangle$ is $r^{j}(1-r)^{n-j}$, and thus, if $a_{j}$ is the number of closed-open sets in $\mathscr{Q}$ whose $\mu(r)$-measure is $r^{j}(1-r)^{n-j}$ (hence $\left.0 \leqslant a_{j} \leqslant n ! / j !(n-j) !\right)$, then

$$
\mu(r)(h(V))=a_{0} r^{n}+a_{1} r^{n-1}(1-r)+\cdots+a_{n}(1-r)^{n} .
$$

Formula (2.4) now follows by substituting into the equation $\mu(s)(V)=$ $\mu(r)(h(V))$ the values obtained for $\mu(s)(V)$ and $\mu(r)(h(V))$. Formula (2.5) is 
proven similarly by considering $h^{-1}$ instead of $h$.

Theorem 2.2 is surely elementary, yet it will prove to be a basic tool in proving the main results which appear in the next section.

3. The main results. Let $K(r)$ denote the class of measures in $\mathscr{F}$ which are topologically equivalent to $\mu(r)$. As was remarked earlier, along with $\mu(r)$ the class $K(r)$ always contains the measure $\mu(1-r)$.

THEOREM 3.1. Each class $K(r)$ contains at most countably many measures of the family $\mathscr{F}$.

Proof. If the measure $\mu(s)$ is to belong to the class $K(r)$, then the number $s$ must satisfy equation (2.4) for some choice of integers $n>1$ and $a_{0}, a_{1}, \ldots, a_{n}$. For fixed $n$, as $0 \leqslant a_{j} \leqslant n ! / j !(n-j) !$, by varying the integers $a_{0}, a_{1}, \ldots, a_{n}$, the right-hand side of (2.4) generates only finitely many different numbers $s$. It follows that the number of measures $\mu(s)$ in the class $K(r)$ is at most countable.

THEOREM 3.2. The number of classes $K(r)$ is the same as the cardinality $c$ of the continuum.

Proof. This result follows immediately from Theorem 3.1 and the fact that the correspondence $r \rightarrow \mu(r)$ is one-to-one.

Theorem 3.2 can be generalized to the family of all Borel measures $\mu$ in $X$. Let $K(\mu)$ denote the class of Borel measures which are topologically equivalent to $\mu$. A Borel measure is determined by its values at basic closed-open sets. As there are only countably many of these, there can only be $c^{\boldsymbol{\alpha}_{0}}=c$ many different Borel measures in $X$. Hence, there are at most $c$ classes $K(\mu)$. However, a class $K(\mu)$ cannot contain different classes $K(r)$, and since there are $c$ of these, there must exist $c$ classes $K(\mu)$. In particular, it follows that there exist $c$ Borel measures in the Cantor space $X$ which are topologically inequivalent to each other.

If $r$ is a rational number and $s$ is any number, both in the unit interval, then the measures $\mu(r)$ and $\mu(s)$ cannot possibly be topologically equivalent unless $s$ is a rational number also. For, indeed, the right-hand side of formula (2.4) defines a rational number whenever $r$ is rational. Thus, if $r$ is rational, then the class $K(r)$ only contains measures $\mu(s)$ with $s$ rational.

The next theorem represents a remarkable and rather surprising result.

TheOREM 3.3. Let $r$ and $s$ be two rational numbers in the unit interval. The measures $\mu(r)$ and $\mu(s)$ are topologically equivalent if and only if $s=r$ or $s=1-r$.

In terms of classes Theorem 3.3 has an equivalent formulation as follows.

THEOREM 3.3'. If $r$ is a rational number in the unit interval, then the class $K(r)$ consists only of the measures $\mu(r)$ and $\mu(1-r)$. 
The proof of Theorem 3.3 is deferred to the next section. In the meantime an example will be used to illustrate the kind of arguments which are involved in the proof. By Theorem 3.3, the measures $\mu\left(\frac{1}{2}\right)$ and $\mu\left(\frac{1}{4}\right)$ cannot be topologically equivalent. Assuming the contrary, Theorem 2.2 makes it possible to write

$$
\frac{1}{2}=b_{0}\left(\frac{1}{4}\right)^{m}+b_{1}\left(\frac{1}{4}\right)^{m-1}\left(\frac{3}{4}\right)+\cdots+b_{m}\left(\frac{3}{4}\right)^{m}
$$

where $b_{0}$ is either 0 or 1 . After clearing denominators (3.1) becomes

$$
2(4)^{m-1}=b_{0}+b_{1} 3+\cdots+b_{m} 3^{m} \text {. }
$$

As $4 \equiv 1(\bmod 3)$, it follows from $(3.2)$ that $2 \equiv b_{0}(\bmod 3)$, which is impossible since $b_{0}$ can only be 0 or 1 .

If $r$ is a transcendental number and $s$ is any irrational number, both in the unit interval, then the measures $\mu(r)$ and $\mu(s)$ cannot be topologically equivalent unless $s$ is transcendental also. For, indeed, the right-hand side of formula (2.5) defines an algebraic number whenever $s$ is algebraic. Thus, if $r$ is transcendental, then the class $K(r)$ only contains measures $\mu(s)$ with $s$ transcendental.

THEOREM 3.4. Let $r$ and $s$ be two transcendental numbers in the unit interval. The measures $\mu(r)$ and $\mu(s)$ are topologically equivalent if, and only if, $s=r$ or $s=1-r$.

Proof. The sufficieny of the condition is clear. To prove that the condition is necessary assume that $\mu(s)$ and $\mu(r)$ are topologically equivalent, and, hence, that formulas (2.4) and (2.5) hold for some choice of integers as stipulated in Theorem 2.2. It follows that $r$ and $s$ can be expressed in the form

$$
\begin{aligned}
& r=r_{0}+r_{1} s+\cdots+r_{j} s^{j}, \\
& s=s_{0}+s_{1} r+\cdots+s_{k} r^{k},
\end{aligned}
$$

where the coefficients $r_{0}, r_{1}, \ldots, r_{j}, s_{0}, s_{1}, \ldots, s_{k}$ are integers with both $r_{0}$ and $s_{0}$ equal to 0 or 1 , where $j$ and $k$ are such that $1<j<m, 1<k<n$, and where $r_{j} \neq 0$ and $s_{k} \neq 0$. Substitute $s$ from (3.4) into (3.3) to obtain, after rearranging terms, a polynomial $p(r)$ in $r$ equal to 0 . As $r$ is transcendental, all the coefficients of $p(r)$ must be zero. If $j k>1$, then the coefficient that goes with $r^{j k}$ is $r_{j}\left(s_{k}\right)^{j}$ which, for as long as $r_{j} \neq 0$ and $s_{k} \neq 0$, cannot be zero. Hence, $j k=1$, which entails that $j=k=1$. It follows that $r=r_{0}+r_{1} s$, $s=s_{0}+s_{1} r$, and, consequently, that

$$
p(r)=r_{0}+r_{1} s_{0}+\left(r_{1} s_{1}-1\right) r .
$$

But then $r_{1} s_{1}-1=0$, so that $r_{1}=s_{1}=1$ or $r_{1}=s_{1}=-1$, and (3.4) becomes (i) $s=s_{0}+r$ or (ii) $s=s_{0}-r$. Since $s=1+r$ is not in the unit interval, $s_{0}$ cannot be 1 in (i). Hence, $s_{0}=0$ and $s=r$. Since $s=-r$ is not in the unit interval, $s_{0}$ cannot be 0 in (ii). Then $s_{0}=1$ and $s=1-r$.

In terms of classes Theorem 3.4 has an equivalent formulation. 
THEOREM 3.4'. If $r$ is a transcendental number in the unit interval, then the class $K(r)$ consists only of the measures $\mu(r)$ and $\mu(1-r)$.

Through Theorem 3.3' and Theorem $3.4^{\prime}$ a definitive intrinsic description of the classes $K(r)$ has been obtained when $r$ is rational or transcendental. The only case that remains open is when $r$ is algebraic, which means that there is complete knowledge of all the classes $K(r)$ except for countably many.

4. Proof of Theorem 3.3. The sufficiency of the condition is clear. To establish that the condition is necessary proceed as follows. Write $r=a / b$ and $s=c / d$, where $b$ and $d$ are positive integers and $a$ and $c$ are nonnegative integers. Assume that the fractions are written in reduced form. By interchanging the roles of $r$ and $s$, if necessary, it may be assumed, without loss of generality, that $b \leqslant d$.

If $r=0$ or 1 , then every closed-open set in $X$ has $\mu(r)$-measure 0 or 1 ; and if $\mu(s)$ and $\mu(r)$ are topologically equivalent, then every closed-open set in $X$ also has $\mu(s)$-measure 0 or 1 . It follows that $s=0$ or 1 . Having disposed of this trivial case, it may be assumed from here on that neither $r$ nor $s$ is $\mathbf{0}$ or $\mathbf{1}$. Hence, $1<b, 0<a<b$ and $0<c<d$.

Suppose from now on that $s \neq r$ and $s \neq 1-r$. It will be shown then that equation (2.5) cannot hold, and hence, that the two measures $\mu(r)$ and $\mu(s)$ must be topologically inequivalent.

Note that if $c=d-c$, then $s=c / d=1 / 2$. Hence, $d=2$, and since $1<b \leqslant d, b=2$ also. But then $a=1$ and $r=1 / 2$. Thus, $c \neq d-c$, and, by interchanging the roles of $s$ and $1-s$, if necessary, it may be assumed, without loss of generality, that $c<d-c$.

It is being assumed throughout that $b \leqslant d$. From here on the proof splits into two cases: case 1 when $b=d$, and case 2 when $b<d$.

Suppose that $b=d$. If it were true that $a=b-a$, then $r=a / b=1 / 2$. Hence, $d=b=2$, and since $0<c<d$, it would follow that $c=1$ and therefore $s=1 / 2$. Thus, $a \neq b-a$ and, by interchanging the roles of $r$ and $1-r$, if necessary, it may be assumed that $a<b-a$. Also, if it were true that $d-c \leqslant a$, then $d-c<b-a$, and as $c<d-c \leqslant a$, it would follow that $d=c+(d-c)<a+(b-a)=b$. But $b=d$. Consequently, $a<d-$ $c$. Finally, observe that under the assumption $b=d$ it is not possible to have $a=c$, as this would entail that $s=r$.

LEMMA 4.1. If $b=d, c<d-c$ and $a<d-c$, then there cannot exist nonnegative integers $b_{0}, b_{1}, \ldots, b_{m}(m \geqslant 1)$ with $b_{0}=0$ or 1 such that

$$
\frac{a}{b}=b_{0}\left(\frac{c}{d}\right)^{m}+b_{1}\left(\frac{c}{d}\right)^{m-1}\left(\frac{d-c}{d}\right)+\cdots+b_{m}\left(\frac{d-c}{d}\right)^{m} .
$$

Proof. Assume that, for some choice of integers, (4.1) holds. Then, upon clearing denominators,

$$
a d^{m-1}=b_{0} c^{m}+b_{1} c^{m-1}(d-c)+\cdots+b_{m}(d-c)^{m} .
$$


As $d \equiv c(\bmod d-c)$, it follows from $(4.2)$ that

$$
a c^{m-1} \equiv b_{0} c^{m} \quad(\bmod d-c) .
$$

Since $c / d$ is in reduced form, the integers $c$ and $d-c$ are relatively prime. Line (4.3) can be divided by $c^{m-1}$ to obtain

$$
a \equiv b_{0} c \quad(\bmod d-c) .
$$

However, $0<a<d-c$, so that it is not possible for $b_{0}$ to be 0 . Thus, $b_{0}=1$, but then $a \equiv c(\bmod d-c)$ is not possible either, as both $a$ and $c$ are less than $d-c$ and $a \neq c$. This contradiction proves the lemma.

Assume now that $b<d$. Then the inequalities $a \geqslant c$ and $b-a \geqslant d-c$ cannot both be true. Thus, $a<c$ or $b-a<d-c$, and there is no loss of generality in assuming that $a<c$, provided that it is not assumed also that $c<d-c$ as was done earlier.

LEMMA 4.2. Let $b<d$ and $a<c$. If $a \equiv b_{m} b(\bmod c)$ and $a \equiv b_{0} b(\bmod d$ - c) with $b_{m}=0$ or $1, b_{0}=0$ or 1 , then (i) $b_{m}=1$, (ii) $a<d-c$, and (iii) $b_{0}=1$.

Proof. If $b_{m}=0$, then $a=k c$ for some positive integer $k$. Hence, $a \geqslant c$, which contradicts the hypothesis. Therefore, $b_{m}=1$, thus proving (i).

It follows from (i) that $b-a=j c$ for some nonnegative integer $j$. As $b \neq a, j$ must be positive. Then $b-a \geqslant c$. If it were true that $a \geqslant d-c$, it would follow that $b=a+(b-a) \geqslant(d-c)+c=d$, or $b \geqslant d$, contrary to the hypothesis. Therefore, $a<d-c$, thus proving (ii). If $b_{0}=0$, then $a=$ $p(d-c)$ for some positive integer $p$. Hence, $a \geqslant d-c$, but this contradicts (ii). So $b_{0}=1$, and the lemma is proved.

Lemma 4.3. Let $b<d$ and $a<c$. Then it is not possible to have $a \equiv b(\bmod$ c) and $a \equiv b(\bmod d-c)$ simultaneously.

Proof. Suppose on the contrary that $b=a+k c$ and that $b=a+j(d-$ c) for some nonnegative integers $k$ and $j$. As $b \neq a$, both $k$ and $j$ must be larger than or equal to 1 . Both inequalities $c<d / 2$ and $d-c<d / 2$ cannot be true simultaneously.

Assume that $c \geqslant d / 2$. If $k \geqslant 2$, then $b=a+k c \geqslant a+d>d$, or $b>d$, which contradicts the hypothesis. Hence, $k=1$. But then, $c=k c=b-a=$ $j(d-c)$, so that $d-c$ divides $c$. But $c$ and $d-c$ are relatively prime and distinct. Hence, $d-c=1$, which is impossible on account of Lemma 4.2(ii) and the fact that $a$ is positive.

It must then be true that $d-c \geqslant d / 2$. If $j \geqslant 2$, then $b=a+j(d-c) \geqslant a$ $+d>d$. But this contradicts the hypothesis. Thus, $j=1$. Then $d-c=j(d$ $-c)=b-a=k c$, and $c$ divides $d-c$. Again, $c$ and $d-c$ are relatively prime and distinct. So, $c=1$, which is impossible as $a<c$ by hypothesis and $a$ is positive. This contradiction proves the lemma. 
LEMMA 4.4. Let $b<d$ and $a<c$. Then there cannot exist nonnegative integers $b_{0}, b_{1}, \ldots, b_{m}(m \geqslant 1)$ with $b_{0}=0$ or $1, b_{m}=0$ or 1 , such that

$$
\frac{a}{b}=b_{0}\left(\frac{c}{d}\right)^{m}+b_{1}\left(\frac{c}{d}\right)^{m-1}\left(\frac{d-c}{d}\right)+\cdots+b_{m}\left(\frac{d-c}{d}\right)^{m} \text {. }
$$

Proof. Assume that, for some choice of integers, (4.5) holds. Then, upon clearing denominators,

$$
d^{m} a=b_{0} b c^{m}+b_{1} b c^{m-1}(d-c)+\cdots+b_{m} b(d-c)^{m} .
$$

As $d \equiv c(\bmod d-c)$ and $d \equiv d-c(\bmod c)$, it follows from $(4.6)$ that

$$
c^{m} a \equiv b_{0} b c^{m} \quad(\bmod d-c)
$$

and

$$
(d-c)^{m} a \equiv b_{m} b(d-c)^{m} \quad(\bmod c) .
$$

Since $c$ and $d-c$ are relatively prime, (4.7) can be divided by $c^{m}$ and (4.8) by $(d-c)^{m}$ to obtain

$$
a \equiv b_{0} b \quad(\bmod d-c)
$$

and

$$
a \equiv b_{m} b \quad(\bmod c)
$$

Then, by Lemma $4.2, b_{0}=b_{m}=1$ and thus $a \equiv b(\bmod c)$ and $a \equiv b$ $(\bmod d-c)$. However, by Lemma 4.3, these two congruences cannot hold simultaneously. This contradiction proves the lemma.

Lemmas 4.1 and 4.4 show that if $s \neq r$ and $s \neq 1-r$, then equation (2.5) does not hold. Consequently, the measures $\mu(r)$ and $\mu(s)$ are not topologically equivalent. Theorem 3.3 has been proved.

ACKNOWLedgement. The results in this article constitute part of the doctoral dissertation presented by the author to the Faculty of Bryn Mawr College. That dissertation was written under the supervision of Professor John C. Oxtoby, whose valuable assistance and kind encouragement the author is pleased to acknowledge.

\section{REFERENCES}

1. J. C. Oxtoby and S. M. Ulam, Measure preserving homeomorphisms and metrical transitivity, Ann. of Math. (2) 42 (1941), 874-920.

2. J. C. Oxtoby, Homeomorphic measures in metric spaces, Proc. Math. Soc. 24 (1970), 419-423.

3. J. C. Oxtoby and V. Prasad, Homeomorphic measures in the Hilbert cube, Pacific J. Math. 77 (1978), 483-497.

4. F. J. Navarro-Bermúdez, Topologically equivalent measures in the Cantor space, $\mathrm{Ph} . \mathrm{D}$. Thesis, Bryn Mawr College, 1977.

Department of Mathematics, Widener College, Chester, Pennsylvania 19013 